\title{
Inicio del tratamiento farmacológico de la tuberculosis
}

\author{
Raúl Cicero-Sabido' y Guillermo Meléndez-Mier²
}

${ }^{1}$ Universidad Nacional Autónoma de México, Facultad de Medicina; ${ }^{2}$ Secretaría de Salud, Hospital General de México, Dirección de Investigación, Ciudad de México, México

La solución de un problema complejo, como la tuberculosis, debe ser vista como un incentivo para desarrollar nuevas maneras para trabajar conjuntamente en beneficio de la comunidad y del hombre mismo.

The Lord hath created medicines out of the Earth; and he that is wise will not abhor them.

[ECCLESIASTICUS, XXXVIII, 4]

\section{Resumen}

La aparición de nuevos fármacos antituberculosos, como la bedaquilina y el delaminid, hace inevitable recordar que los primeros ensayos estrictamente controlados del tratamiento médico de la tuberculosis se publicaron en dos artículos de excelente y rigurosa investigación científica que pueden calificarse como históricos. En 1948 se estudió formalmente la estreptomicina como medicamento antituberculoso eficaz. En 1952, en otro ensayo se comparó estreptomicina-ácido paraaminosalicílico con isoniacida, con lo que se establecieron las primeras bases del tratamiento farmacológico de la tuberculosis.

PALABRAS CLAVE: Estreptomicina. Isoniacida. Ácido paraaminosalicílico. Farmacorresistencia.

\begin{abstract}
The appearance of new anti-tuberculosis drugs such as bedaquiline and delamanid makes it impossible not to remember that the first strictly controlled medical trials of tuberculosis treatment were published in two rigorously researched outstanding articles that can be qualified as historical. In 1948, streptomycin was formally studied as an efficacious anti-tuberculosis drug. In 1952, another trial compared streptomycin-paramino salicylic acid with isoniazid, by means of which the first bases of pharmacological tuberculosis treatment were established.
\end{abstract}

KEY WORDS: Streptomycin. Isoniazid. Para-aminosalicylic acid. Drug-resistance.

Ante la aparición de nuevos fármacos antituberculosos como la bedaquilina y el delaminid ${ }^{1}$ es importante recordar que los primeros ensayos estrictamente controlados del tratamiento médico de la tuberculosis se publicaron en dos artículos de excelente y rigurosa investigación científica que pueden calificarse como históricos. El descubrimiento de la estreptomicina por Selman Waksman en 1944 y su empleo como el primer medicamento antituberculoso eficaz se estudió formalmente en $1948^{2}$ y fue seguido en 1952 por otro ensayo que comparaba estreptomicina-ácido paraaminosalicílico con isoniacida, ${ }^{3}$ con lo que se establecieron las primeras bases del tratamiento farmacológico (quimioterapia) de la tuberculosis. Al mismo tiempo se observó la aparición de la drogorresistencia desarrollada por $M y$ cobacterium tuberculosis, que constituye un reto terapéutico.
Fecha de recepción: 31-07-2017

Fecha de aceptación: 10-08-2017

DOI: 10.24875/GMM.17003616
Gac Med Mex. 2018;154:620-621

Disponible en PubMed www.gacetamedicademexico.com 
En el primer ensayo de 1948 participaron 11 expertos de siete importantes hospitales, coordinados por Geoffrey Marshall del Brompton Hospital de Londres, quienes diseñaron el protocolo sobre los efectos de la estreptomicina en la tuberculosis pulmonar al comparar el reposo en cama con la administración del fármaco: murió un número importante de pacientes con la primera medida. De quienes recibieron estreptomicina, muchos presentaron considerable mejoría clínica y radiológica a los tres meses, si bien en otros casos se encontró que el bacilo había desarrollado resistencia al antibiótico en diferentes tiempos del tratamiento. Esta investigación fue avalada por el British Medical Research Council como ejemplo de ensayo con resultados valorados con métodos estadísticos de selección aleatoria. ${ }^{4}$

Un 1952 se publicó "TREATMENT of pulmonary tuberculosis with isoniazid; an interim report to the Medical Research Council by their Tuberculosis Chemotherapy Trials Committee", en el que se reportaron los resultados de la investigación coordinada por Marc Daniels y Wallace Fox, en la que se compararon isoniacida y estreptomicina-ácido paraaminosalicílico. En este ensayo se concluyó que la isoniacida sola no es más efectiva que la estreptomicina-ácido paraaminosalicílico y se encontró que $71 \%$ de las cepas de Mycobacterium tuberculosis se volvió resistente al cabo de tres meses de tratamiento.

La realización de estos dos protocolos fue trascendental en la lucha contra la tuberculosis; indicaron que la combinación de varias drogas podría lograr la curación. La combinación de isoniacida, rifampicina, pirazinamida y etambutol es la base del tratamiento antituberculoso estrictamente supervisado que se emplea actualmente en todo el mundo. La resistencia, multifarmacorresistencia y resistencia extrema persisten como un verdadero reto para lograr la curación de la tuberculosis.
Cada nuevo fármaco antituberculoso debe ser sometido a un estricto protocolo, incluso en animales de experimentación, para demostrar su eficiencia y determinar la susceptibilidad de Mycobacterium tuberculosis y si este presenta resistencia que pueda anular el efecto terapéutico del medicamento. La aprobación por parte de la Organización Mundial de la Salud y de la Food and Drugs Administration es vital para la aplicación clínica y comercialización de cualquier fármaco. ${ }^{5}$

En la tuberculosis no solo el descubrimiento y empleo de nuevas drogas son esenciales, sino trabajar por un mundo donde todos sus habitantes tengan acceso a los servicios sanitarios, con programas de lucha antituberculosa estrictamente realizados con fármacos eficaces, y donde se abata pobreza, ${ }^{6}$ que está ligada a la tuberculosis y dificulta la detección y tratamiento de la enfermedad. ${ }^{7}$ Por la grave desigualdad que priva en diversos países, ${ }^{7}$ la erradicación de la tuberculosis es tal vez una utopía. ${ }^{7}$ La búsqueda de nuevos medicamentos eficaces debe continuar como una esperanza para los pacientes infectados con cepas de micobacterias resistentes. El ejemplo de quienes lo han hecho está presente.

\section{Bibliografia}

1. Bayfield A, editor. Martindale. The complete drug reference. Pharmaceutical Press: Londres; 2014.

2. Streptomycin treatment of pulmonary tuberculosis. A medical research council investigation. Br Med J. 1948;2(4582):769-782.

3. TREATMENT of pulmonary tuberculosis with isoniazid; an interim report to the Medical Research Council by their Tuberculosis Chemotherapy Trials Committee. Br Med J. 1952;2(4787):735-746.

4. Chalmers I. Why the 1948 MRC trial of streptomycin used treatment allocation based on random numbers. J R Soc Med. 2011;104(9):383-386.

5. Michinson D, Davies G. The chemotherapy of tuberculosis: past, present and future. Int J Tuberc Lung Dis. 2012;16(6):724-732.

6. World Health Organization. Global tuberculosis report 2015. Suiza: World Health Organization; 2016.

7. Ross A, Rosenberg P, Dye C, Prasad A. A world cities and the end of TB. Trans R Soc Trop Med Hyg. 2016;110(3):151-152. 